\title{
An additional term of the Galactic tide due to dark matter (Research Note)
}

\author{
L. Neslušan \\ Astronomical Institute, Slovak Academy of Sciences, 05960 Tatranská Lomnica, Slovakia \\ e-mail: ne@ta3.sk
}

Received 29 July 2011 / Accepted 12 September 2011

\begin{abstract}
Aims. We aim to complete the formula characterizing the Galactic tide acting on the comets in the Oort Cloud with the term that describes the action of dark matter in the Galaxy.

Methods. We analytically derive this term and gauge its amplitude for the current Galactic location of the Sun.

Results. The amplitude of the new component of the tide, which acts in the radial direction from the Galactic centre, is estimated to be about $15 \%$ lower than that of the old, commonly considered radial component, so it should not be neglected in a precise calculations. And, since it depends on the distance $r$ from the Galactic centre as $r^{-2}$, it should certainly be considered when the Oort-Cloud dynamics is studied on a long-time scale assuming a radial migration of the Sun to shorter galactocentric distances.
\end{abstract}

Key words. solar neighborhood - Galaxy: kinematics and dynamics - Oort Cloud - celestial mechanics

\section{Introduction}

The Galactic tide has been found to be the strongest outer perturber of the comet orbits in the Oort Cloud during its recent dynamical history. The dominant component of the tide is the action of matter concentrated in the Galactic disc. Every object is more attracted by a larger bulk of this matter in the direction toward the disc plane than in the opposite direction, away from the disc. Another force is caused by the motion of the Solar System around the Galactic centre. Due to this motion along a curved trajectory, the galactic heliocentric coordinate system is not inertial. Because of its rotation, a centrifugal force occurs on a long-period scale.

The acceleration from the Galactic tide used to be described in the modified rectangular galactic coordinate system, $O \tilde{x} \tilde{y} \tilde{z}$, with the $\tilde{x}$-axis oriented outward from the Galactic centre, the $\tilde{y}$-axis parallel to the Galactic plane and oriented in the sense of Galactic rotation, and the $\tilde{z}$-axis oriented toward the South Galactic Pole. In this coordinate system, the corresponding rectangular components of the acceleration are (Heisler \& Tremaine 1986)

$a_{\tilde{x}}=(A-B)(3 A+B) \tilde{x}$,

$a_{\tilde{y}}=-(A-B)^{2} \tilde{y}$,

$a_{\tilde{z}}=-\left[4 \pi k^{2} \rho-2\left(B^{2}-A^{2}\right)\right] \tilde{z}$,

where $A$ and $B$ are the Oort's constants characterizing the Galactic rotation, $k$ is the Gauss' gravitational constant, and $\rho$ the density of Galactic matter in solar neighbourhood.

At present, we know that a larger part of gravitating matter is in the form of dark matter. Its gravitational attraction is commonly taken into account when the motion of the Sun or other star(s) in the Galaxy is numerically integrated. Some examples can be found, e.g., in the recently published monograph by Binney \& Tremaine (2008) where the complex description of the Galactic gravitational potential, including the dark matter, is also presented. The part of the monograph concerning our work is based mainly on earlier works by Dehnen \& Binney (1998) and Binney \& Merrifield (1998).

Although the gravitational characteristics of the dark matter in the Galaxy are known, more or less, these characteristics have not been considered in the studies of the dynamics of comets in the Oort Cloud. In this short contribution, we derive, present, and thus propagate the new term of the Galactic tide that has to exist because the action of the dark matter. The significance of this term is discussed in Sect. 3.

\section{The tide from the dark matter in the Galaxy}

The force from the dark matter inside the Galaxy is evident from the Galactic-rotation curve. Because of the interstellar absorption, the whole rotation curve for our Galaxy is not as well determined observationally as the rotation curves of neighbouring spiral galaxies. Nevertheless, we can expect that the basic features of all these curves are similar and, therefore, also exist in our own Galaxy.

The known rotation curves of 25 galaxies of various Hubble types can be seen, for example, in the work by Bosma (1978, Chap. 6.4, Fig. 1). Seeing these or other examples of rotational velocity behaviour, we can conclude that the velocity only weakly depends on $r$ for a wide interval of larger galactocentric distances, including the current distance of the Sun from the 
Galactic centre. Consequently, the correspodingly derived behaviour of mass, $M(r)$, inside the galactocentric sphere of radius $r$, increases almost linearly with $r$. (A good example can be seen in Bosma 1978, Chap. 6.4, Fig. 3.)

We are aware of a suggestion that this linear approximation is sufficient for the function $M=M(r)$ in a larger interval of $r$, especially in more precise calculations. Now that we have mentioned this approximation, we just want to justify it in a relatively short interval of $r$ comparable to the known diameter of the Oort Cloud, which is $\sim 1 \mathrm{pc}$ (cf. with the distance of the Sun from the Galactic centre equal to $\sim 8 \mathrm{kpc}$ ), where it can most probably be regarded as sufficient.

That the nature of dark matter remains unknown could represent a potential problem. It is possible that the dark matter emerged from the early Universe with some scale-free distribution of density fluctuations. The fluctuations led to a hierarchically aggregating population of dark matter haloes (White \& Rees 1978; White \& Frenk 1991). The formation of these structural patterns was simulated, e.g., within the Aquarius Project (Springel et al. 2008). The authors detected four generations of subhaloes within subhaloes. If a subhalo was situated in a vicinity of the Solar System, its action on the Oort Cloud would be more like a perturbation to a single passing star than due to a smooth distribution of matter and, therefore, would largely invalidate our result. The probability of a subhalo occurring near the Solar System is, however, very low. Springel et al. predict the mass fraction in a dark-matter substructure to be below $0.1 \%$ within the solar circle. In addition, the existence of structured dark matter is still questionable. Some other authors (e.g. Colombi 2008) have drawn an opposite conclusion that most of the detectable signal from dark matter in our Milky Way probably comes from the main, smooth Galactic halo, rather than from small clumps.

In our derivation of the new term, we consider a more or less smooth distribution of dark matter to which the Oort-Cloud comets are sensitive and assume, as one possibility, the linear approximation of the mass $M(r)$ inside the sphere of radius $r$. This mass can, in this case, be given as

$M(r)=C_{\mathrm{o}} r$,

where $C_{\mathrm{o}}$ is a constant of proportionality. The radial (i.e. in the direction of $\tilde{x}$-axis) acceleration of the Sun caused by the matter of mass $M$ is

$a_{\odot}=-\frac{k^{2} M}{r^{2}}=-\frac{k^{2} C_{0}}{r}$.

Similarly, the acceleration of a comet nucleus in the galactocentric distance $r+\tilde{x}$ (so, $\tilde{x}$ is the radial component of the nucleus' heliocenric distance) is

$a_{\mathrm{cn}}=-\frac{k^{2} M}{(r+\tilde{x})^{2}}=-\frac{k^{2} C_{\mathrm{o}}}{r+\tilde{x}}$.

Since the Sun has, most probably, never been in close vicinity to the Galactic centre, it has always been valid that $\tilde{x} \ll r$. Using this inequality, the last relation can be simplified to

$a_{\mathrm{cn}}=-\frac{k^{2} C_{\mathrm{o}}}{r}\left(1-\frac{\tilde{x}}{r}\right)$.

The heliocentric, i.e. tidal, acceleration of the nucleus is the difference, $\Delta a_{\mathrm{cn}}$, between the galactocentric accelerations of both nucleus and Sun:

$\Delta a_{\mathrm{cn}}=a_{\mathrm{cn}}-a_{\odot}=\frac{k^{2} C_{\mathrm{o}} \tilde{x}}{r^{2}}$.
Constant $C_{\mathrm{o}}$ can be gauged by considering the current galactocentric distance of the Sun $R_{\mathrm{O}}=(8.0 \pm 0.5) \mathrm{kpc}$ and corresponding mass $M\left(R_{\mathrm{O}}\right) \equiv M_{\mathrm{O}}=9.0 \times 10^{10} M_{\odot}$ (Binney \& Tremaine 2008, p. 18). Mass $M_{\mathrm{o}}$ is calculated for the circular velocity equal to $v_{\text {circ }}=(220 \pm 20) \mathrm{km} \mathrm{s}^{-1}$ at the above value of $R_{\mathrm{o}}$. With the help of $R_{\mathrm{o}}$ and $M_{\mathrm{o}}$, constant $C_{\mathrm{o}}=M_{\mathrm{o}} / R_{\mathrm{o}}$, according to relation (4). Subsequently, the new tidal term (8) can be rewritten as

$\Delta a_{\mathrm{cn}}=\frac{k^{2} M_{\mathrm{o}} \tilde{x}}{R_{\mathrm{o}} r^{2}}$

for whatever distance $r$ (but where the linear approximation (4) remains valid).

For the current Oort Cloud, the identity $r=R_{\mathrm{O}}$ is true, therefore the entire $\tilde{x}$-term of the Galactic tide can be given as

$a_{\tilde{x} \text {,new }}=(A-B)(3 A+B) \tilde{x}+\frac{k^{2} M_{\mathrm{o}}}{R_{\mathrm{o}}^{3}} \tilde{x}$

\section{(cf. relation (1)).}

If the function $M=M(r)$ for an arbitrary $r$ is not approximated by the linear behaviour, but is calculated according to a model of Galactic gravitational potential, the new term must also be considered in the form of $k^{2} M \tilde{x} / r^{3}$.

\section{Concluding remarks}

If we consider the density of Galactic matter in solar vicinity $\rho=0.10 M_{\odot} \mathrm{pc}^{-3}$ (Holmberg \& Flynn 2000), then the ratio of amplitudes of both dominant $\tilde{z}$-term and dark-matter $\tilde{x}$-term equals $4 \pi k^{2} \rho /\left(k^{2} M_{\mathrm{o}} / R_{\mathrm{o}}^{3}\right)=7.1$. For the values of the Oort's constants $A=(14.8 \pm 0.8) \mathrm{km} \mathrm{s}^{-1} \mathrm{kpc}^{-1}$ and $B=-(12.4 \pm$ 0.6) $\mathrm{km} \mathrm{s}^{-1} \mathrm{kpc}^{-1}$ (Feast \& Whitelock 1997; see also Binney \& Tremaine 2008, p. 18), the ratio of the known centrifugal $\tilde{x}$-term and new dark-matter $\tilde{x}$-term is 1.15 . Therefore, the amplitude of the new term is only about $\sim 15 \%$ lower than the old $\tilde{x}$-term. It is the smallest term of all, but is not negligible in more precise calculations for the current location of the Sun in the Galaxy.

In the Oort-Cloud dynamical studies in the past, a radial excursion of the Sun has been considered (Matese et al. 1995; Brasser et al. 2010). Because of the $a_{\tilde{x} \text {,new }} \propto r^{-2}$ dependence, the new term becomes more significant in shorter galactocentric distances of the Sun and its comet cloud. For example, Brasser et al. (2010) consider the solar motion from 2 to $20 \mathrm{kpc}$ from the Galactic centre. In the distance of $2 \mathrm{kpc}$, which is four times shorter than the current distance, the amplitude of the new term is about 16 times larger. At the same time, the currently dominant $\tilde{z}$-term is weaker in $2 \mathrm{kpc}$ (Brasser et al. 2010, Fig. 4), therefore the new term is clearly dominant here. The total amplitude of the tide is larger in $2 \mathrm{kpc}$ than in $8 \mathrm{kpc}$, just because of the new term.

Although the intrinsic nature of the dark matter is unknown, its gravitational effect has been well mapped observationally, and it is certain that this effect does exist. As mentioned in Sect. 2, it is common to take it into account in various investigations of stellar trajectories in the Galaxy, so it should also be included in more precise calculations of perturbations of OortCloud-comet orbits.

Acknowledgements. Part of this work was supported by the Slovak Research and Development Agency (grant No. SK-FR-0004-09). 


\section{References}

Binney, J., \& Merrifield, M. 1998, Galactic Astronomy (Princeton: Princeton Univ. Press)

Binney, J., \& Tremaine, S. 2008, Galactic Dynamics, 2nd ed. (Princeton and Oxford: Princeton Univ. Press)

Bosma, A. 1978, The distribution and kinematics of neutral hydrogen in spiral galaxies of various morphological types, Ph.D. Thesis, Groningen: Rijksuniversiteit Groningen

Brasser, R., Higuchi, A., \& Kaib, N. 2010, A\&A, 516, A72
Colombi, S. 2008, Nature, 456, 44

Dehnen, W., \& Binney, J. 1998, MNRAS, 294, 429

Feast, M., \& Whitelock, P. 1997, MNRAS, 291, 683

Heisler, J., \& Tremaine, S. 1986, Icarus, 65, 13

Holmberg, J., \& Flynn, C. 2000, MNRAS, 313, 209

Matese, J. J., Whitman, P. G., Innanen, K. A., \& Valtonen, M. J. 1995, Icarus, 116,255

Springel, V., Wang, J., Vogelsberger, M., et al. 2008, MNRAS, 391, 1685

White, S. D. M., \& Frenk, C. S. 1991, ApJ, 379, 52

White, S. D. M., \& Rees, M. J. 1978, MNRAS, 183, 341 